\title{
Strain and Thermally Induced Magnetic Dynamics and Spin Current in Magnetic Insulators Subject to Transient Optical Grating
}

\author{
Xi-Guang Wang*, Levan Chotorlishvili and Jamal Berakdar* \\ Institute of Physics, Martin Luther University Halle-Wittenberg, Halle, Germany
}

We analyze the magnetic dynamics and particularly the spin current in an opencircuit ferromagnetic insulator irradiated by two intense, phase-locked laser pulses. The interference of the laser beams generates a transient optical grating and a transient spatiotemporal temperature distribution. Both effects lead to elastic and heat waves at the surface and into the bulk of the sample. The strain induced spin current as well

OPEN ACCESS

Edited by:

Daniel Schick,

Helmholtz-Zentrum Berlin (HZ),

Germany

Reviewed by:

Raanan Tobey,

University of Groningen, Netherlands Anna Marina Pandolfi,

Politecnico di Milano, Italy

*Correspondence:

$X i$-Guang Wang

wangxiguangsss@gmail.com;

Jamal Berakdar

jamal.berakdar@physik.uni-halle.de

Specialty section:

This article was submitted to

Mechanics of Materials,

a section of the journal

Frontiers in Materials

Received: 19 April 2017 Accepted: 22 June 2017

Published: 14 July 2017

Citation:

Wang X-G, Chotorlishvili L and Berakdar J (2017) Strain and

Thermally Induced Magnetic

Dynamics and Spin Current in

Magnetic Insulators Subject to

Transient Optical Grating.

Front. Mater. 4:19.

doi: 10.3389/fmats.2017.00019 as the thermally induced magnonic spin current are evaluated numerically on the basis of micromagnetic simulations using solutions of the heat equation. We observe that the thermo-elastically induced magnonic spin current propagates on a distance larger than the characteristic size of thermal profile, an effect useful for applications in remote detection of spin caloritronics phenomena. Our findings point out that exploiting strain adds a new twist to heat-assisted magnetic switching and spin-current generation for spintronic applications.

Keywords: magnonic spin current, optical grating, elastic wave, laser pulses, micromagnetic simulations, spintronics, caloritronics

\section{INTRODUCTION}

Magneto-elastic coupling (Kittel, 1958; Akhiezer et al., 1959) enables the manipulation of the magnetization via elastic means. Fueled by potential applications in spintronics and sensorics thermal and magnetoelastic driven magnetic dynamics is attacking a great deal of research (Davis et al., 2010; Scherbakov et al., 2010; Roy et al., 2011; Uchida et al., 2011a; Weiler et al., 2011; Azovtsev and Pertsev, 2016). Elastic excitations might be triggered in a variety of ways, for instance via acoustic transducers (Davis et al., 2010; Uchida et al., 2011b; Weiler et al., 2011, 2012; Thevenard et al., 2013) or by optical methods (Scherbakov et al., 2010; Kim et al., 2012; Jäger et al., 2013; Kovalenko et al., 2013; Afanasiev et al., 2014). Optical methods mainly generate elastic excitations via laser heating and thermoelastic effects. As compared to transducers, laser pulses achieve larger amplitude thermoelastic deformations (Saito et al., 2003; Scherbakov et al., 2010; Kim et al., 2012; Jäger et al., 2013; Kovalenko et al., 2013; Afanasiev et al., 2014). A further interesting method to induce magnetoelastic excitations via electric fields is to use multiferroics materials, particularly heterostructures of coupled piezoelectric or ferroelectric and ferromagnetic layers (Vaz, 2012; Cherepov et al., 2014; Jia et al., 2014, 2016; Gilbert et al., 2016). In recent experiments (Janušonis et al., 2015, 2016), a transient optical grating is generated on the surface of a ferromagnet by two phase-locked interfering laser pulses. On the length scale of the interference pattern, a frequency tunable elastic wave is generated. By virtue of magneto-elastic coupling, a magnetization precession in the ferromagnetic 
film is launched. This method bridges the transducer and the optical approaches. Hitherto, these experiments were interpreted on the basis of elastically driven magnetic precession without thermal fluctuations. Obviously, for the laser intensities generating efficient optical grating heating effects are unavoidable and may affect the magnetization dynamics. In fact, the thermal magnetization dynamics generated by a single laser pump has been studied recently. It has been found that the laser-induced thermal gradient generates a propagating thermal magnonic spin current (magnonic spin Seebeck effect) (Schreier et al., 2013; Roschewsky et al., 2014; Etesami et al., 2015; Giles et al., 2015). Hence, we expect that a transient optical grating may bring about new phenomena in addition to the aforementioned magnetoelastic waves, particularly the magnonic spin Seebeck effect will be inspected below. For the magnetization simulations, we use classical micromagnetic theory. The magnonic spin Seebeck is studied under the influence of the transient optical grating and in the preence of magneto-elastic excitations triggered by the elastic waves. At the surface of the ferromagnetic film, we analyze the effects of thermal gradient and surface elastic wave excitation on the magnonic spin current. The elastic wave excites resonantly the magnetization dynamics and magnonic spin current near the ferromagnetic resonance frequency. This prediction is in line with the recent experiments reported by Janušonis et al. (2015, 2016). Furthermore, we identify a magnonic spin current flowing perpendicular to the film surface plane, i.e., perpendicular to the propagation direction of the elastic waves. The spin current is induced by the thermal gradient away from the laser spots into the depth of the sample. The elastic wave excitation is shown to be very weak in this direction. In addition, changing the magnetization direction the effect of the magneto-elastic coupling can be suppressed. The diffusion length of the magnonic spin current and the effect of the pulse length are discussed in light of the obtained results.

\section{RESULTS AND DISCUSSIONS}

\subsection{Materials and Methods}

We consider an experimental situation consisting of a transient optical grating generated by the interference of two laser beams impinging on the surface plane $(x-y$ plane) of an insulating ferromagnet (cf. Figure 1). The laser heating results in a timedependent non-uniform temperature profile $[T(x, y, z, t)]$. Due to thermoelastic effects, the laser heating triggers an elastic wave at the surface of the thick ferromagnetic film. The thermal fluctuations and the elastic excitations cooperatively contribute to the magnetization dynamics and generate a propagating magnon flow. To study the magnetization dynamics, we utilize the stochastic Landau-Lifshitz-Gilbert equation including thermal fluctuations and magneto-elastic coupling effects

$$
\frac{\partial \mathbf{M}}{\partial t}=-\gamma \mathbf{M} \times\left(\mathbf{H}_{e f f}+\mathbf{h}\right)+\frac{\alpha}{M_{s}} \mathbf{M} \times \frac{\partial \mathbf{M}}{\partial t}
$$

where $\mathbf{M}$ is the magnetization of the sample, $M_{s}$ is the saturation magnetization, $\gamma$ is the gyro-magnetic ratio, and $\alpha$ is the phenomenological Gilbert damping constant. The total effective field $\mathbf{H}_{\text {eff }}$ consists of the exchange field $\frac{2 A_{e x}}{\mu_{0} M_{s}^{2}} \Delta \mathbf{M}$, the external

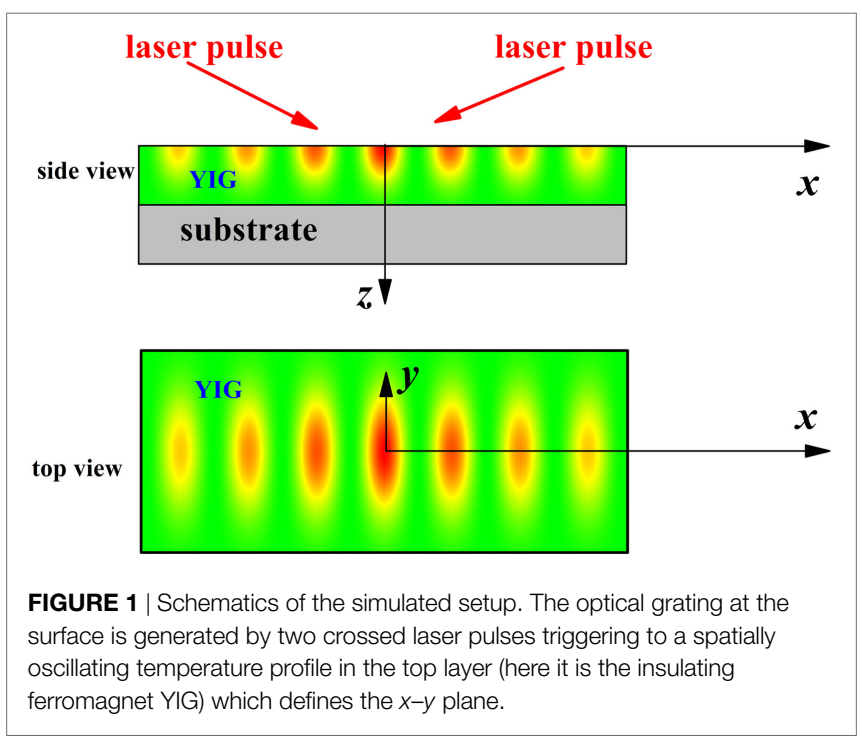

field $H_{\text {ext }}$ aligned along $x$ axis, and the magneto-elastic coupling field $\mathbf{H}_{m e}$. The random field $\mathbf{h}$ stems from thermal fluctuations (García-Palacios and Lázaro, 1998)

$$
\left\langle h_{i}(t, \mathbf{r}) h_{j}\left(t^{\prime}, \mathbf{r}^{\prime}\right)\right\rangle=\frac{2 k_{B} T \alpha}{\gamma M_{S} V} \delta_{i j} \delta\left(\mathbf{r}-\mathbf{r}^{\prime}\right) \delta\left(t-t^{\prime}\right)
$$

here, $T(\mathbf{r}, t)$ is the temperature profile formed due to laser heating, $k_{B}$ is the Boltzmann constant and $V$ is the volume of the sample.

$[T(x, y, z, t)]$ derives from the solution of the heat equation (Etesami et al., 2015):

$$
\frac{\partial T(x, y, z, t)}{\partial t}=\frac{k_{p h}}{\rho C} \nabla^{2} T(x, y, z, t)+I(x, y, z, t)
$$

$k_{p h}$ is the thermal conductivity, $\rho$ is the mass density, and $C$ is the heat capacity. The source term $I(x, y, z, t)$ signifies a spatially periodic laser intensity generated by the transient grating, i.e.,

$$
I(x, y, z, t)=I_{0}(t) \cos ^{2}\left(2 \pi x / r_{l}\right) e^{-\delta_{T}|z|} e^{-\lambda^{2}\left(x^{2}+y^{2}\right)}
$$

$\delta_{T}$ is the laser penetration depth and $\lambda$ is the inverse radius of the radial beam. We implement periodic series of the rectangular laser pulses $S\left(2 \pi f_{\text {mode }} t\right)$ with the modulation frequency $f_{\text {mode }}=0.1 \mathrm{GHz}$. Here, $I_{0}(t)=\frac{\delta_{T}}{\rho \mathrm{C}} I_{00} S\left(2 \pi f_{\text {mode }} t\right)$, and $I_{00}$ is the intensity of the pulse. The periodic function $\cos ^{2}\left(2 \pi x / r_{l}\right)$ describes the effect of the transient grating. The grating period $r_{l} / 2$ is tunable by the phase difference between the two laser beams (Etesami et al., 2015; Janušonis et al., 2015, 2016). The profile of laser intensity $I$ is shown in Figure 2 for $r_{l}=2.8 \mu \mathrm{m}$. The oscillating behavior of $I$ along the $x$ axis is related the transient grating. Apparently $I$ decays exponentially along the $z$ axis (perpendicular to the surface).

The laser beams are such that they generate a transient grating which is oscillating along the $x$ direction. The temperature profile $T$ along the $x$ axis is also spatially oscillating (numerical results are shown below), and decays along the $z$ axis (which is perpendicular 


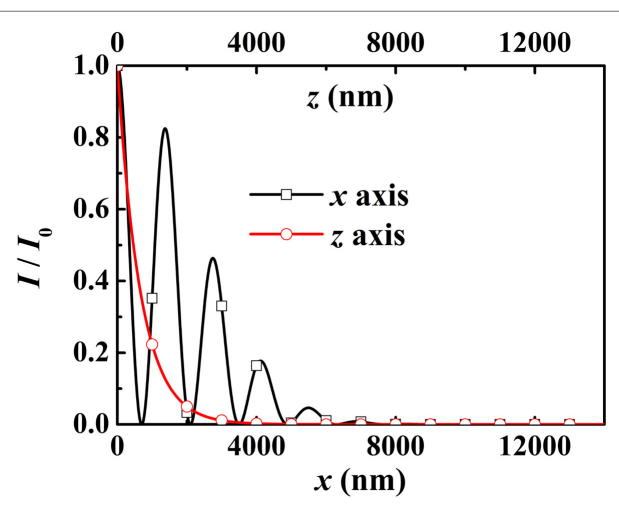

FIGURE 2 | Profiles of the normalized laser intensity $I / l_{0}$ along $x$ axis $(y=0$ and $z=0)$ and $z$ axis $(x=0$ and $y=0)$.

to the surface) into the bulk of the sample. The oscillating heat profile generates a periodic elastic deformation and a propagating elastic wave along the $x$ axis. For simplicity, we use the propagating wave solution $u_{\mathrm{amp}} \sin (\omega t-k x)$ to describe the excited elastic waves in our simulation, and $u_{\mathrm{amp}}$ is the amplitude. Here, $\omega=2 \pi f$ is the wave frequency, and $k$ is the wave vector. The wave is only located in the film surface due to the form of the temperature profile (Janušonis et al., 2015, 2016). The wave-vector is determined by the period of the transmitted grating $k=4 \pi / r_{l}$, and the frequency is a function of the wave-vector $f=v \Lambda$ with $v \approx 5,600 \mathrm{~m} / \mathrm{s}$. The elastic amplitude $u_{\mathrm{amp}}$ increases linearly with the laser intensity $I_{00}$.

The magneto-elastic coupling term $\mathbf{H}_{m e}=-\frac{1}{\mu_{0} M_{s}} \delta F_{m e} / \delta \mathbf{m}$ derives from the magneto-elastic energy $F_{m e}$ (Akhiezer et al., 1959; Azovtsev and Pertsev, 2016),

$$
\begin{aligned}
F_{m e}= & b_{1}\left(e_{x x} m_{x}^{2}+e_{y y} m_{y}^{2}+e_{z z} m_{z}^{2}\right) \\
& +b_{2}\left(e_{x y} m_{x} m_{y}+e_{x z} m_{x} m_{z}+e_{y z} m_{y} m_{z}\right)
\end{aligned}
$$

here, $b_{1}$ and $b_{2}$ are material dependent magneto-elastic coupling constants, and $e_{x x}, e_{x y} \ldots$ are the components of the strain tensor. For simplicity, only the $e_{x z}$ component is considered. Although $e_{x x}$ is found to be large (Janušonis et al., 2015, 2016), it does not contribute to the magnetization dynamics for the equilibrium magnetization along the $x$ axis (a strong enough external field $H_{\text {ext }}$ is applied along the $x$ axis).

In the modeling of the magnetization dynamics in the ferromagnet, we use the material parameters of YIG (Table 1). For simplicity, two one-dimensional models are considered for two cases: (i) the magnetization dynamics in the film surface is solved in a model along $x$ axis for $y=0$ and $z=0$ with the surface propagating elastic wave. Here, the component $e_{x z}=\frac{1}{2} \frac{U_{z}}{x}=\varepsilon \sin (\omega t-k x)$ is calculated as the spatial derivative of the displacement $U_{z}$ in the $z$ direction, where $\varepsilon$ is a constant. For $b_{2} \varepsilon=200 \mathrm{~J} / \mathrm{m}^{3}$, the amplitude of $U_{z}$ is about $0.15 \mathrm{~nm}$. (ii) in the direction perpendicular to the surface, the model along $z$ axis for $x=0$ and $y=0$ is calculated without elastic wave. The external field $H_{\text {ext }}=1.14 \times 10^{5} \mathrm{~A} / \mathrm{m}$ is applied along the $x$ axis and the equilibrium magnetization $\mathbf{m}_{0}$ is in the $+x$ direction. For the laser profile (Eq. 4), we use $f_{\text {mode }}=0.1 \mathrm{GHz}$ and $I_{00}=2 \times 10^{10} \mathrm{~W} / \mathrm{m}^{2}$.
TABLE 1 | Parameters for YIG.

\begin{tabular}{llll}
\hline & Definition & Value & Unit \\
\hline$M_{\mathrm{S}}$ & Saturation magnetization & $1.4 \times 10^{5}$ & $\mathrm{~A} / \mathrm{m}$ \\
$A_{\mathrm{ex}}$ & Exchange constant & $5 \times 10^{-12}$ & $\mathrm{~J} / \mathrm{m}$ \\
$\alpha$ & Damping constant & 0.005 & \\
$\rho$ & Mass density & 5170 & $\mathrm{~kg} / \mathrm{m}^{3}$ \\
$C$ & Heat capacity & 570 & $\mathrm{~J} /(\mathrm{kg} \mathrm{K})$ \\
$k_{\text {ph }}$ & Thermal conductivity & 0.02 & $\mathrm{~W} /(\mathrm{m} \mathrm{K})$ \\
$\delta_{T}$ & Laser penetration depth & $1.5 \times 10^{6}$ & $\mathrm{~m}^{-1}$ \\
$\lambda$ & Inverse radial beam radius & $\sqrt{1 \times 10^{11}}$ & $\mathrm{~m}^{-1}$ \\
$\gamma$ & Gyro-magnetic ratio & $2.21 \times 10^{5}$ & $\mathrm{~m} /\left(\mathrm{Ass}^{-2}\right.$ \\
$\mu_{0}$ & Permeability & $4 \pi \times 10^{-7}$ & $\mathrm{~N} / \mathrm{A}^{-2}$ \\
$k_{\mathrm{B}}$ & Boltzmann constant & $1.3806 \times 10^{-23}$ & $\mathrm{~N} / \mathrm{A}^{-2}$ \\
$b_{2}$ & Magneto-elastic coupling constant & 0.55 & $\mathrm{MJ} / \mathrm{m}^{3}$ \\
\end{tabular}

\subsection{Model (i) Along $x$ Axis}

In the film surface, the spatially oscillating temperature profile for $r_{l}=2.8 \mu \mathrm{m}$ is generated as shown in Figure 3. The temperature $T$ increases linearly with time when $S\left(2 \pi f_{\text {mode }} t\right) \neq 0$, and it remains nearly unchanged in the region of a zero laser intensity $\left(S\left(2 \pi f_{\text {mode }} t\right)=0\right)$. For $0<t<50 \mathrm{~ns}$, the spatial profile of $T(x)$ is always spatially oscillating. We note that in reality the temperature increase by laser heating is limited. With heating up the sample the thermal losses due to the environment increases. As the time span of our simulations is limited to only $50 \mathrm{~ns}$, this limiting factor of temperature increase is not accounted for. The elastic wave has the wave-vector $k=4 \pi / r_{l}$ and the frequency $f=4 \mathrm{GHz}$. Solving Eq. 1, the magnonic spin current $J_{x}=\frac{2 \gamma A_{e x}}{\mu_{0} M_{s}^{2}}\left(M_{y} \partial_{x} M_{z}-M_{z} \partial_{x} M_{y}\right)$ propagating along the $x$ axis is calculated. Here, $M_{y}$ and $M_{z}$ are the $y$ and $z$ components of the local magnetization, respectively. The numerical results are statistically averaged over 1,000 realizations of the noise. Apart from boundary effects, the spatial profile of $J_{x}(x)$ is always constant, as shown in Figure $\mathbf{4 A}$, signifying a steady current. In the time domain (Figure 4B), after an initial increase and oscillations, $J_{x}(t)$ attains the steady state constant value. Varying the grating period $r_{l}$, the features of the magnonic spin current $J_{x}$ change, as shown in Figure $5\left(r_{l}=3.7 \mu \mathrm{m}\right.$ and $f=3 \mathrm{GHz}$ ). The value of $J_{x}$ becomes much smaller compared to that for $f=4 \mathrm{GHz}$. Also, the spatial profile of $J_{x}(x)$ turns spatially oscillating after $t=1 \mathrm{~ns}$, and it approaches 0 at $x=0$ due to the free boundary conditions. Besides, similar to the time-dependent temperature $T(t)$, the current $J_{x}(t)$ increases gradually with time $t$, and oscillates with a frequency of $0.1 \mathrm{GHz}$.

The $r_{l}$-dependent phenomena above are ascribed to a magnetization resonance behavior. Based on the spin-wave dispersion relation $2 \pi f_{s w}=\frac{2 \gamma A_{e x}}{\mu_{0} M_{s}} k_{s w}^{2}+\gamma H_{e x t}$, the resonance frequency is estimated to be $4 \mathrm{GHz}$ when the spin wave vector $k_{s w}$ is equal to the wave vector $2 \pi \Lambda$ of the propagating elastic wave, i.e., the resonance condition. Here, we note that the influence of the dynamic magneto-elastic coupling on the spin-wave dispersion relation is weak enough to be neglected. At the resonance frequency $f=4 \mathrm{GHz}$, the spin-wave amplitude excited by the elastic wave is very large, and dominates the value of $J_{x}$. As the time independent amplitude of the elastic wave is not spatially varying, $J_{x}$ is independent of distance $x$ and time $t$ (Figure 4). For a different frequency $(f=3 \mathrm{GHz})$ away from the resonance condition, the spin-wave amplitude excited by the elastic wave becomes smaller 

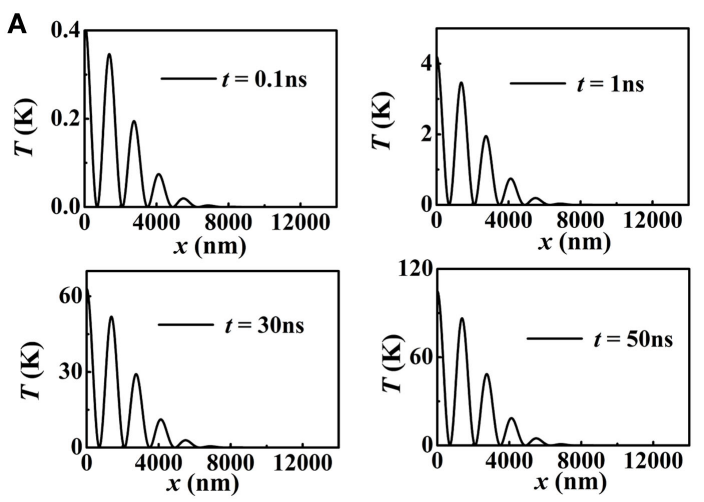

B

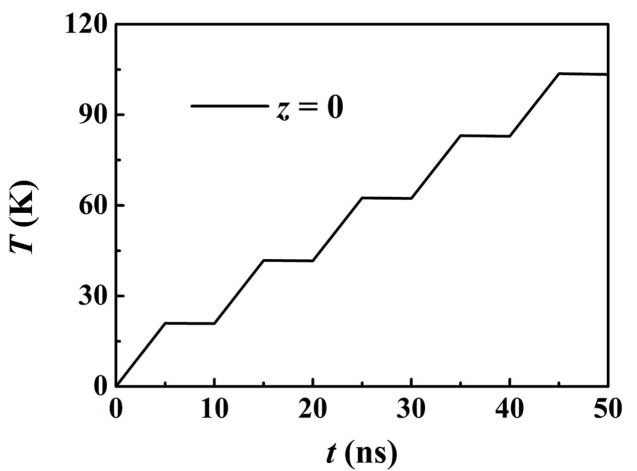

FIGURE 3 | (A) The spatiotemporal temperature profiles of $T(x, t)$ at $t=0.1,1,30$, and 50 ns. (B) At $x=0$, temperature $T$ as a function of time $t$.
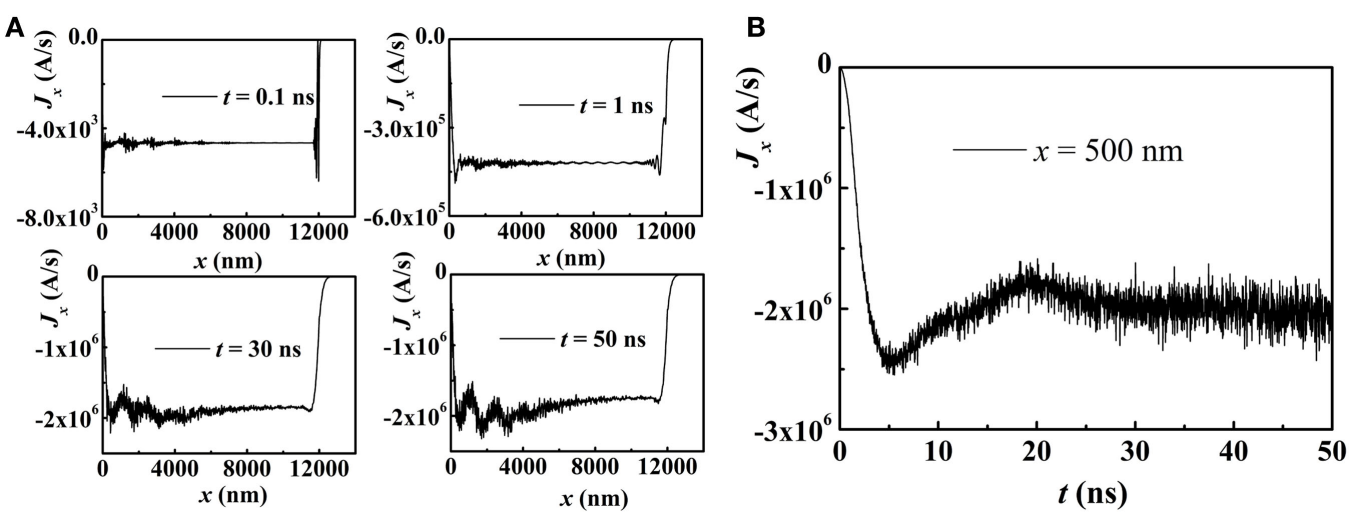

FIGURE $4 \mid$ For $r_{l}=2.8 \mu \mathrm{m}$ and $f=4 \mathrm{GHz}$, (A) the profiles of the magnonic spin current $J_{x}(x)$ at $t=0.1,1,30$, and $50 \mathrm{~ns}$. (B) At $x=500 \mathrm{~nm}$, $J_{x}$ as a function of time $t$. The equilibrium magnetization is in the $+x$ direction.
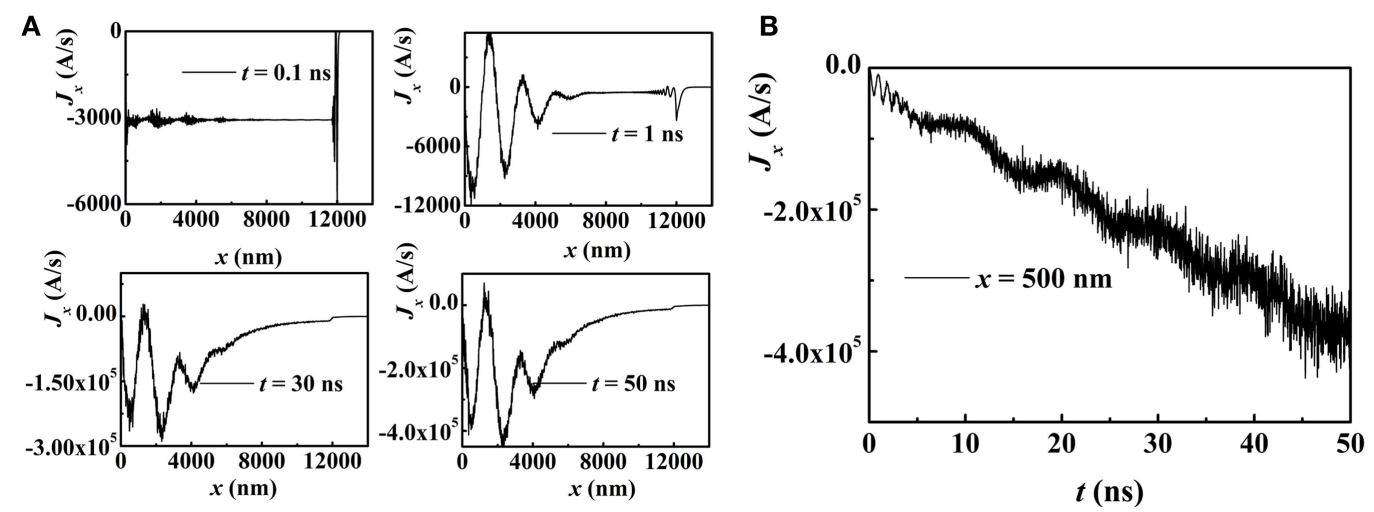

FIGURE 5 | For $r_{l}=3.7 \mu \mathrm{m}$ and $f=3 \mathrm{GHz}$, (A) the profiles of the magnonic spin current $J_{x}(x)$ at $t=0.1,1,30$, and $50 \mathrm{~ns}$. (B) At $x=500 \mathrm{~nm}, J_{x}$ as a function of time $t$. The equilibrium magnetization $\mathbf{m}_{0}$ is in the $+x$ direction.

than the thermal effect. The thermal gradient dominates the value of $J_{x}$, and $J_{x}$ varies with $x$ and time $t$.

To detect the effect of a thermal gradient, we apply an external field $H_{\text {ext }}=1.14 \times 10^{5} \mathrm{~A} / \mathrm{m}$ along $y$ axis. The equilibrium magnetization $\mathbf{m}_{0}$ is in the $+y$ direction. In this case, the magneto-elastic coupling field $\mathbf{H}_{m e}$ is very small, and the magnonic spin current $J_{x}$ is dominated by the thermal gradient. As shown in Figure 6, $J_{x}=\frac{2 \gamma A_{e x}}{\mu_{0} M_{s}^{2}}\left(M_{z} \partial_{x} M_{x}-M_{x} \partial_{x} M_{z}\right)$ gradually increases with the temperature and has an oscillatory profile along the $x$ axis. 

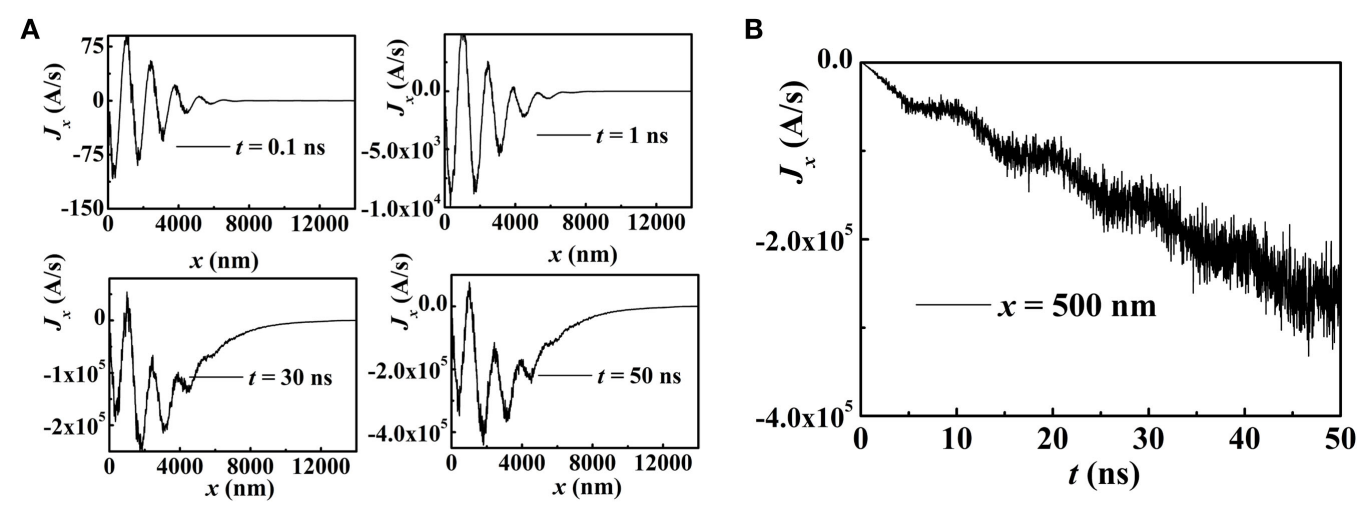

FIGURE 6 | For $r_{l}=2.8 \mu \mathrm{m}$ and $f=4 \mathrm{GHz}$, (A) the profiles of the magnonic spin current $J_{x}(x)$ at $t=0.1,1,30$, and $50 \mathrm{~ns}$. (B) At $x=500 \mathrm{~nm}, J_{x}$ as a function of time $t$. The equilibrium magnetization $\mathbf{m}_{0}$ is in the $+y$ direction.
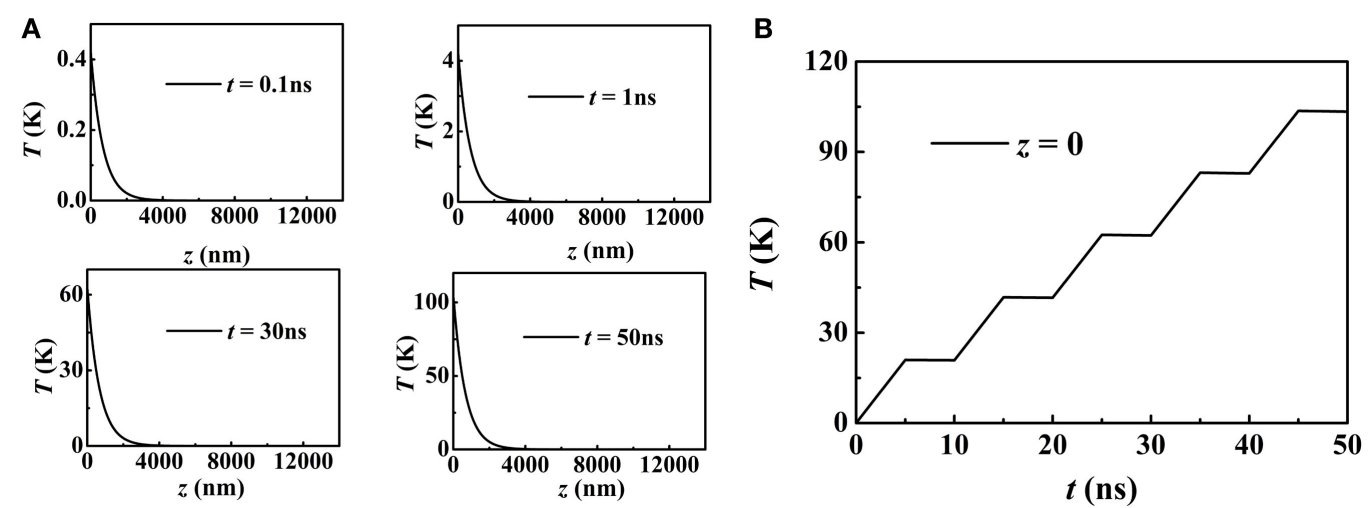

FIGURE $7 \mid$ (A) The profiles of $T(z, t)$ at $t=0.1,1,30$, and $50 \mathrm{~ns}$. (B) At $z=0$, temperature $T$ as a function of time $t$

\subsection{Model (ii) Along z Axis}

The optical grating is periodic in the $x$ direction only. For the effects on the spin dynamics along the $z$ direction (perpendicular to the surface), we solve Eq. 3 with the laser intensity being $I$ decaying with $z$ (Figure 2). The temperature profile $T(z)$ is similar to $I(z)$, as shown in Figure 7A. Also, in Figure 7B, the temperature increases linearly with $t$ in the region of $I \neq 0$. In this model, the generated magnonic spin current $J_{z}=\frac{2 \gamma A_{e x}}{\mu_{0} M_{s}^{2}}\left(M_{y} \partial_{z} M_{z}-M_{z} \partial_{z} M_{y}\right)$ propagates along the $z$ axis and is shown in Figure 8. Here, the equilibrium magnetization is in the $+x$ direction. Excited by the thermal gradient, $J_{z}(z)$ reaches its maximum near the boundary. The zero spin current density at $x=0$ is caused by the boundary effect. With time, $J_{z}(t)$ has a similar profile with $T(t)$ increasing gradually in the oscillation frequency of $0.1 \mathrm{GHz}$. Moreover, the above features are independent of the equilibrium magnetization $\mathbf{m}_{0}$. By changing the equilibrium magnetization $\mathbf{m}_{0}$ to the $+y$ direction, the values of magnonic spin current remain unchanged (not shown).

\subsection{Spin Current As a Surface Heat Sensor}

Our numerical results indicate that the diffusion length of thermal magnonic spin current can be larger than that of the thermal profile. To show this effect, the thermal magnonic spin current
$J_{x}(x)$, the thermal profile $T(x)$ and the laser profile $I / I_{0}$ are shown together in Figure 9A. Here, the model (i) is adopted, and the equilibrium magnetization $\mathbf{m}_{0}$ in the $+y$ direction is used to remove the effect of elastic waves. The features of the thermal profile $T(x)$ are completely determined by $I / I_{0}$ but $J_{x}(x)$ is different. Apart from boundary effects, $J_{x}(x)$ decays exponentially with $x$, together with the oscillations caused by $I / I_{0}$. We use a non-linear function $J_{0} \cos ^{2}\left(2 \pi x / r_{l}\right) e^{-\lambda^{2} x^{2}}+J_{1} e^{-\delta_{S}^{x}|x|}$ for fitting the curve of $J_{x}$ in $x>1000 \mathrm{~nm}$ (Figure 9A), and $\delta_{S}^{x}=2.45 \times 10^{5}$ $\mathrm{m}^{-1}$ is obtained. Obviously, the thermal magnonic spin current $J_{x}(x)$ diffuses longer than the region where the temperature $T(x)$ changing. The same effect exists also for the model (ii) along $z$ axis, as shown in Figure 9B. Here, the non-linear fitting function $J_{0} e^{-\delta_{T}|z|}+J_{1} e^{-\delta_{S}^{z}|z|}$ is adopted, and $\delta_{S}^{z}=5.9 \times 10^{5} \mathrm{~m}^{-1}$ is obtained. $\delta_{S}^{z}$ is obviously smaller than $\delta_{T}$, indicating a larger diffusion length $1 / \delta_{S}^{z}$ of $J_{z}$. This difference in the range of the heat current and the spin current can be utilized to sense a temperature increase at the surface by measuring the associated spin current, e.g., via the inverse spin Hall effect.

\subsection{Duration of the Laser Pulse}

The effect of the duration of the laser pulse in a single period is also of relevance. The duration $5 \mathrm{~ns}$ is used in the above 

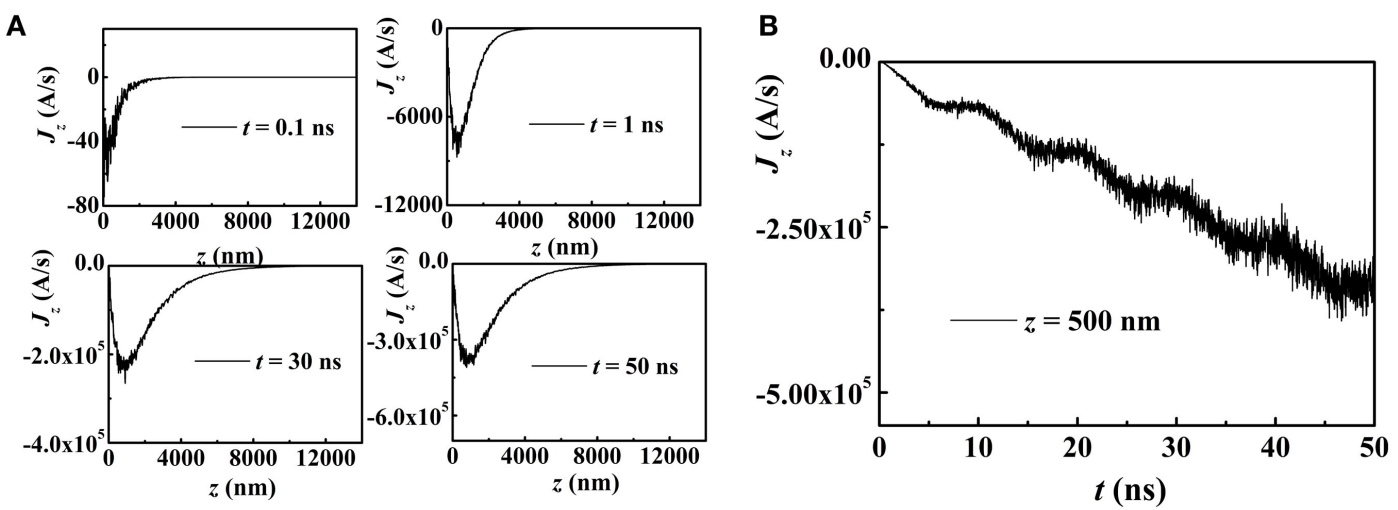

FIGURE 8 | (A) The profiles of the magnonic spin current $J_{z}(z)$ at $t=0.1,1,30$, and $50 \mathrm{~ns}$. (B) At $z=500 \mathrm{~nm}, J_{z}$ as a function of time $t$. The equilibrium magnetization $\mathbf{m}_{0}$ is in the $+x$ direction.
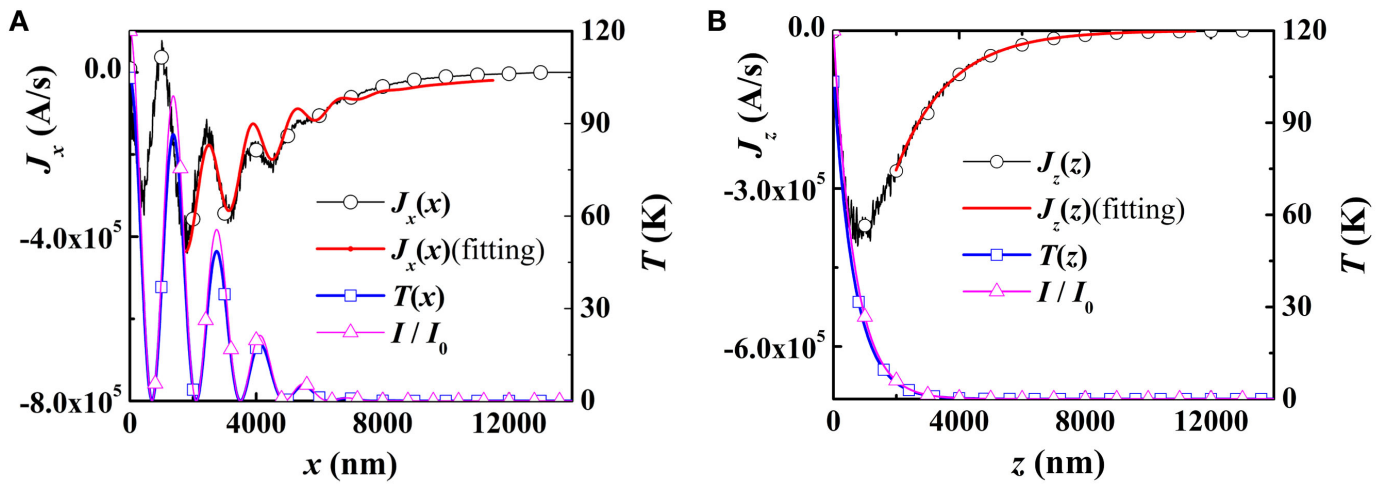

FIGURE 9 | At $t=50 \mathrm{~ns}$, (A) profiles of the magnonic spin current $J_{x}$ (simulations and fitting curves), temperature $T(x)$, and laser intensity $I / I_{0}$. (B) Profiles of the magnonic spin current $J_{z}$ (simulations and fitting curves), the temperature $T(z)$, and the laser intensity $I / I_{0}$.
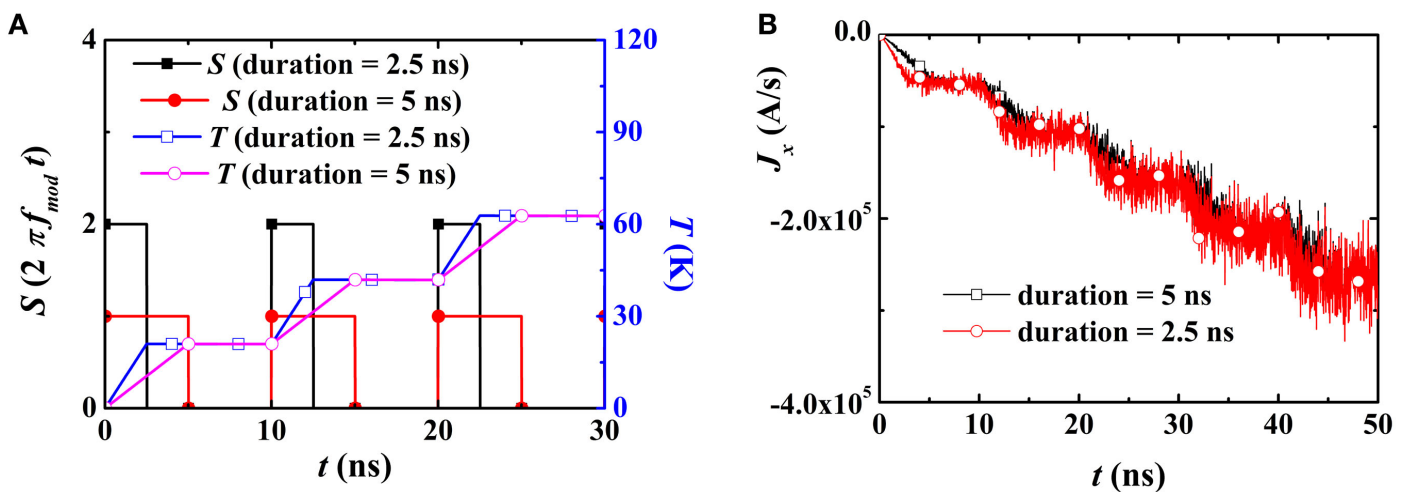

FIGURE 10 ( (A) Time-varying periodic rectangular pulses $S\left(2 \pi f_{\text {mode }} t\right)$ and $T(x=0, t)$. The duration of non-zero $S$ in a single period is $2.5 \mathrm{~ns}$ (squares) and $5 \mathrm{~ns}$ (circles). (B) At $x=500 \mathrm{~nm}, J_{x}$ as a function of time $t$ for the duration of 2.5 and $5 \mathrm{~ns}$.

simulations, as demonstrated by the periodic rectangular pulse $S\left(2 \pi f_{\text {mode }} t\right)$ in Figure 10A. By setting $f_{\text {mode }}=0.1 \mathrm{GHz}$ and changing the duration to $2.5 \mathrm{~ns}$, we find that the temperature increase in a single period is unchanged. Here, the amplitude of $S$ is twice larger than that for duration of $5 \mathrm{~ns}$, to keep the energy pumped in a period unchanged. Finally, at $t=50 \mathrm{~ns}$, the temperature profiles for a duration of $2.5 \mathrm{~ns}$ are still the same as for the curves shown in Figures 3A and 7A. The corresponding magnonic spin current has the same feature, as shown in Figure 10B. 


\subsection{Conclusion}

To conclude, for a magnetic film surface, we considered the magnonic spin current excited by thermal effects and by elastic waves generated by an optical transient grating is studied. Approaching the ferromagnetic resonance, the magnonic spin current is triggered dominantly by the laser-generated elastic wave. Away from resonance, thermal effects dominate. By manipulating the equilibrium magnetization, one can totally suppress the effects of elastic waves. The magnonic spin current excitation perpendicular to the film surface is also studied and found to be determined by thermal effects only. Besides, we find the thermal magnonic spin current to diffuse to regions well beyond the spatial extension of the temperature gradient generated by the laser pulse. This effect provides a new method to detect thermal effects remotely on a long range scale.

\section{REFERENCES}

Afanasiev, D., Razdolski, I., Skibinsky, K. M., Bolotin, D., Yagupov, S. V., Strugatsky, M. B., et al. (2014). Laser excitation of lattice-driven anharmonic magnetization dynamics in dielectric $\mathrm{FeBo}_{3}$. Phys. Rev. Lett. 112, 147403. doi:10.1103/ PhysRevLett.112.147403

Akhiezer, A., Bar'iakhtar, V., and Peletminskii, S. (1959). Coupled magnetoelastic waves in ferromagnetic media and ferroacoustic resonance. J. Exp. Theor. Phys. $8,157$.

Azovtsev, A. V., and Pertsev, N. A. (2016). Magnetization dynamics and spin pumping induced by standing elastic waves. Phys. Rev. B 94, 184401. doi:10. 1103/PhysRevB.94.184401

Cherepov, S., Amiri, P. K., Alzate, J. G., Wong, K., Lewis, M., Upadhyaya, P., et al. (2014). Electric-field-induced spin wave generation using multiferroic magnetoelectric cells. Appl. Phys. Lett. 104, 082403. doi:10.1063/1.4865916

Davis, S., Baruth, A., and Adenwalla, S. (2010). Magnetization dynamics triggered by surface acoustic waves. Appl. Phys. Lett. 97, 232507. doi:10.1063/1.3521289

Etesami, S. R., Chotorlishvili, L., and Berakdar, J. (2015). Spectral characteristics of time resolved magnonic spin Seebeck effect. Appl. Phys. Lett. 107, 132402. doi:10.1063/1.4931701

García-Palacios, J. L., and Lázaro, F. J. (1998). Langevin-dynamics study of the dynamical properties of small magnetic particles. Phys. Rev. B 58, 14937-14958. doi:10.1103/PhysRevB.58.14937

Gilbert, I., Chavez, A. C., Pierce, D. T., Unguris, J., Sun, W.-Y., Liang, C.-Y., et al. (2016). Magnetic microscopy and simulation of strain-mediated control of magnetization in PMN-PT/Ni nanostructures. Appl. Phys. Lett. 109, 162404. doi:10.1063/1.4965028

Giles, B. L., Yang, Z., Jamison, J. S., and Myers, R. C. (2015). Long-range pure magnon spin diffusion observed in a nonlocal spin-Seebeck geometry. Phys. Rev. B 92, 224415. doi:10.1103/PhysRevB.92.224415

Jäger, J. V., Scherbakov, A. V., Linnik, T. L., Yakovlev, D. R., Wang, M., Wadley, P., et al. (2013). Picosecond inverse magnetostriction in galfenol thin films. Appl. Phys. Lett. 103, 032409. doi:10.1063/1.4816014

Janušonis, J., Chang, C. L., Jansma, T., Gatilova, A., Vlasov, V. S., Lomonosov, A. M., et al. (2016). Ultrafast magnetoelastic probing of surface acoustic transients. Phys. Rev. B 94, 024415. doi:10.1103/PhysRevB.94.024415

Janušonis, J., Chang, C. L., van Loosdrecht, P. H. M., and Tobey, R. I. (2015). Frequency tunable surface magneto elastic waves. Appl. Phys. Lett. 106, 181601. doi:10.1063/1.4919882

Jia, C., Zhang, N., Sukhov, A., and Berakdar, J. (2016). Ultrafast transient dynamics in composite multiferroics. New J. Phys. 18, 023002. doi:10.1088/1367-2630/18/ 2/023002

Jia, C.-L., Wei, T.-L., Jiang, C.-J., Xue, D.-S., Sukhov, A., and Berakdar, J. (2014). Mechanism of interfacial magnetoelectric coupling in composite multiferroics. Phys. Rev. B 90, 054423. doi:10.1103/PhysRevB.90.054423

Kim, J.-W., Vomir, M., and Bigot, J.-Y. (2012). Ultrafast magnetoacoustics in nickel films. Phys. Rev. Lett. 109, 166601. doi:10.1103/PhysRevLett.109.166601

Kittel, C. (1958). Interaction of spin waves and ultrasonic waves in ferromagnetic crystals. Phys. Rev. J. Arch. 110, 836-841. doi:10.1103/PhysRev.110.836

\section{AUTHOR CONTRIBUTIONS}

$\mathrm{X}-\mathrm{GW}$ conducted all numerical calculations and provided the initial draft of the manuscript. LC performed the analytical part. JB conceived and supervised the project. All authors contributed to the interpretation and writing of the paper.

\section{ACKNOWLEDGMENTS}

We thank Dr. S. R. Etesami for useful discussions and consultations.

\section{FUNDING}

This research was funded by the German Science Foundation (DFG) under SFB 762.

Kovalenko, O., Pezeril, T., and Temnov, V. V. (2013). New concept for magnetization switching by ultrafast acoustic pulses. Phys. Rev. Lett. 110, 266602 doi:10.1103/PhysRevLett.110.266602

Roschewsky, N., Schreier, M., Kamra, A., Schade, F., Ganzhorn, K., Meyer, S., et al. (2014). Time resolved spin Seebeck effect experiments. Appl. Phys. Lett. 104, 202410. doi:10.1063/1.4879462

Roy, K., Bandyopadhyay, S., and Atulasimha, J. (2011). Hybrid spintronics and straintronics: a magnetic technology for ultra low energy computing and signal processing. Appl. Phys. Lett. 99, 063108. doi:10.1063/1.3624900

Saito, T., Matsuda, O., and Wright, O. B. (2003). Picosecond acoustic phonon pulse generation in nickel and chromium. Phys. Rev. B 67, 205421. doi:10.1103/ PhysRevB.67.205421

Scherbakov, A. V., Salasyuk, A. S., Akimov, A. V., Liu, X., Bombeck, M., Brüggemann, C., et al. (2010). Coherent magnetization precession in ferromagnetic (Ga,Mn)As induced by picosecond acoustic pulses. Phys. Rev. Lett. 105, 117204. doi:10.1103/PhysRevLett.105.117204

Schreier, M., Kamra, A., Weiler, M., Xiao, J., Bauer, G. E. W., Gross, R., et al. (2013). Magnon, phonon, and electron temperature profiles and the spin Seebeck effect in magnetic insulator/normal metal hybrid structures. Phys. Rev. B 88, 094410. doi:10.1103/PhysRevB.88.094410

Thevenard, L., Duquesne, J.-Y., Peronne, E., von Bardeleben, H. J., Jaffres, H., Ruttala, S., et al. (2013). Irreversible magnetization switching using surface acoustic waves. Phys. Rev. B 87, 144402. doi:10.1103/PhysRevB.87.144402

Uchida, K., Adachi, H., An, T., Ota, T., Toda, M., Hillebrands, B., et al. (2011a) Long-range spin Seebeck effect and acoustic spin pumping. Nat. Mater. 10, 737-741. doi:10.1038/nmat3099

Uchida, K., An, T., Kajiwara, Y., Toda, M., and Saitoh, E. (2011b). Surface-acousticwave-driven spin pumping in $\mathrm{Y}_{3} \mathrm{Fe}_{5} \mathrm{O}_{12} /$ Pt hybrid structure. Appl. Phys. Lett. 99, 212501. doi: $10.1063 / 1.3662032$

Vaz, C. A. F. (2012). Electric field control of magnetism in multiferroic heterostructures. J. Phys. Condens. Matter 24, 333201. doi:10.1088/0953-8984/ $24 / 33 / 333201$

Weiler, M., Dreher, L., Heeg, C., Huebl, H., Gross, R., Brandt, M. S., et al. (2011) Elastically driven ferromagnetic resonance in nickel thin films. Phys. Rev. Lett. 106, 117601. doi:10.1103/PhysRevLett.106.117601

Weiler, M., Huebl, H., Goerg, F. S., Czeschka, F. D., Gross, R., and Goennenwein, S. T. B. (2012). Spin pumping with coherent elastic waves. Phys. Rev. Lett. 108, 176601. doi:10.1103/PhysRevLett.108.176601

Conflict of Interest Statement: The authors declare that the research was conducted in the absence of any commercial or financial relationships that could be construed as a potential conflict of interest.

Copyright (C) 2017 Wang, Chotorlishvili and Berakdar. This is an open-access article distributed under the terms of the Creative Commons Attribution License (CC BY). The use, distribution or reproduction in other forums is permitted, provided the original author(s) or licensor are credited and that the original publication in this journal is cited, in accordance with accepted academic practice. No use, distribution or reproduction is permitted which does not comply with these terms. 